\title{
Deletion of P2 promoter of GJB1 gene a cause of Charcot-Marie-Tooth disease
}

${ }^{1} \mathrm{R}$ Kulshrestha, ${ }^{2} \mathrm{~S}$. Burton-Jones, ${ }^{3} \mathrm{~T}$. Antoniadi, ${ }^{4} \mathrm{M}$ Rogers, ${ }^{5} \mathrm{Z}$. Jaunmuktane, ${ }^{5} \mathrm{~S}$ Brandner,

${ }^{1} \mathrm{~N}$ Kiely, ${ }^{6} \mathrm{R}$. Manuel, ${ }^{1} \mathrm{~T}$ Willis

1. Robert Jones and Agnes Hunt Orthopaedic Hospital, Oswestry

2. University Hospital Bristol NHS Trust, Bristol

3. West Midlands Molecular Genetics Lab, Birmingham

4. Cardiff and Vale UHB - Medical Genetics

5. UCL Institute of Neurology, London

6. Royal Stoke University Hospital, Newcastle Road, Stoke-on-Trent

Corresponding author:

Richa Kulshrestha

Robert Jones and Agnes Hunt Orthopaedic Hospital

Gobowen

Oswestry

SY10 7AG

Phone: 01691404378

Email: richakulshrestha@nhs.net 


\begin{abstract}
X-linked Charcot-Marie-Tooth disease (CMT) is the second most common cause of CMT, and is usually caused by mutations in the gap junction protein beta $1(G J B 1)$ gene. This gene has nerve specific $\mathrm{P} 2$ promoter that work synergistically with $S O X 10$ and EGR2 genes to initiate transcription. Mutation in this region is known to cause Schwann cell dysfunction. A single large family of $\mathrm{X}$ linked peripheral neuropathy was identified in our practice. Next generation sequencing for targeted panel assay identified an upstream exon-splicing deletion identified extending from nucleotide c.-5413 to approximately - c.-49. This matches the sequence of 32 nucleotides at positions c.*218-*249 in the 3'UTR downstream of the GJB1 gene. The deleted fragment included the entire P2 promoter region. The deletion segregated with the disease. To our knowledge a deletion of the P2 promoter alone as a cause of CMT has not been reported previously.
\end{abstract}

\title{
Highlights:
}

- Report of novel mutation as a cause of CMT.

- Complete deletion of P2 promoter region of GJB1 gene.

- Supporting the observation that P2 promoter is central for Schwann cell function.

Key words: Charcot Marie Tooth disease; X-linked; GJB1; connexin 32; deletion; P2 promoter; Schwann cell dysfunction.

Abbreviations: CMT: Charcot-Marie-Tooth; GJB1: gap junction beta 1; CX32: connexin 32; SOX10: SRY-Box 10; EGR2: early growth response 2; IPN: inherited peripheral neuropathy; NGS: next generation sequencing; 


\section{Introduction}

$\mathrm{X}$ linked Charcot-Marie-Tooth (CMT) disease is second most common cause of CMT caused by mutation in gap junction beta $1(G J B 1)$ gene which code for connexin 32 (CX32).[1] Clinically patients present with distal muscle weakness, wasting and sensory loss.[2] The connexin 32 messenger RNA and protein are expressed in Schwann cells in peripheral nervous system,[3] confined to Schmidt-Lantermann incisures and paranodal segment of nodes of Ranvier.[1] GJB1 gene has nerve tissue specific P2 promoter [4,5] that is crucial in initiating transcription.[6] Mutations in the P2 promoter have been reported to cause $\mathrm{X}$ linked CMT suggesting its impairment leading to Schwann cell dysfunction. $[7,8]$ The loss of connexin 32 expression is secondary to failed activation of its promoter by SOX1O and EGR2 which work synergistically for GJB1 gene transcription.[9] In this case series we report a novel mutation of $\mathrm{P} 2$ promoter region of $G J B 1$ gene segregating with the disease.

\section{Patients and Method}

In our practice we identified a large family of $X$ linked inherited peripheral neuropathy with unconfirmed molecular genetics. Genomic DNA was extracted from peripheral blood according to standard methods. Analysis of 56 genes associated with inherited peripheral neuropathy (IPN) was achieved using a custom-designed next generation sequencing (NGS) targeted panel assay. Raw data analysis and filtering was performed using a bespoke opensource pipeline with UCSC hg19 human genome as the reference. Basic copy number enumeration was performed using the CONTRA tool as a component of the analysis pipeline.[10] Clinical details including onset of symptoms, signs, neurophysiology and histology features of affected family members were obtained from the case records with the consent of participants for this publication. 


\section{Results}

\subsection{Genetics}

In the female index patient no pathogenic sequence variants were detected on testing for panel genes including SOX10 and EGR2. However on review, target coverage analysis of the gene panel data indicated a possible heterozygous partial deletion in the $G J B 1$ gene region. This deletion was evident on MLPA dosage analysis13 using MRC Holland probe mix P405A1, which showed abnormal dosage of a single probe, indicating a heterozygous deletion within or including the exon 1B (non-coding) region of the GJB1 gene at Xq13.1, and corresponding to the P2 promoter. Direct PCR (using primers flanking the deletion break points) and Sanger sequence analysis was subsequently used to confirm and characterise this deletion further. The identified deletion in the proband removed 5365 nucleotides and extends from position c.-5413 (5769 bases upstream of the ATG start codon) to nucleotide c.49 (405 bases upstream of the ATG) inclusive. Additionally, a 32 base pair sequence is inserted between these break points; a BLAST search [http://blast.ncbi.nlm.nih.gov/Blast.cgi] indicated that this matches the sequence of 32 nucleotides at positions c. $* 218$ * 249 in the 3 ' untranslated region, downstream of the $G J B 1$ gene. Sanger sequence analysis has revealed that it is likely this sequence is duplicated and inserted between the break points. The deleted fragment in this patient (c.-5413_-49) includes the entire P2 promoter (figure 1). This deletion is confirmed to be present in all affected family members.

\subsection{Clinical features}

Affected individuals showed clinical evidence of a length dependent peripheral neuropathy. Clinical features of affected individuals were variable. They had clawing of toes, feet deformity (cavovarus and plano valgus), wasting of small muscles of hand, (figure 2) absent reflexes and a glove and stocking distribution of sensory involvement. Clinical features are 
summarized in table 1. Males had earlier onset of symptoms than females. Neuropathy was inherited in $\mathrm{X}$ linked dominant fashion with no male-to-male transmission (figure 3). Five affected family members had neurophysiology assessment. They had sensorimotor demyelinating (four patients) and axonal (one patient) neuropathy. Nerve conduction velocities were considerably slowed $(<38 \mathrm{~m} / \mathrm{sec})$, suggesting serious demyelinating component in four patients (table 2). They did not have central nervous system features hence magnetic resonance imaging of brain and spine was not performed.

\subsection{Histology}

The sural nerve biopsy was assessed in one patient by light microscopy and ultrastructuraly by electron microscopy (figure 4). Immunohistochemical (IHC) shows prominent loss of large myelinated fibres (IHC for myelin basic protein, SMI94 antibody), whilst unmyelinated and small myelinated fibres are better preserved (immunostaining for neurofilaments, SMI31 antibody). Ultrastructural examination highlights the regeneration clusters but shows no signs of active macrophage-associated demyelination or chronic demyelinating/re-myelinating process. The nerve biopsy excluded inflammation, showed chronic axonal neuropathy with no evidence of inflammatory vasculitic process.

\section{Discussion}

GJB1 gene, a common cause of X linked CMT maps to chromosome Xq13.1 and is $10 \mathrm{~kb}$ in length. This gene encodes 238- aminoacid gap junction, beta 1/connexin 32 protein that is involved to form hemichannel connexons as diffusion pathway between Schwann cell nucleus and axon.[11] The gene has nerve specific P2 promoter,[4,5] coding region (exon 2) and three non-coding exons (1,1A and 1B). Pathogenic mutations are described in both coding and non-coding region of the gene (http://www.molgen.ua.ac.be/CMTMutations/). Similarly pathogenic variants in P2 promoter region have been reported.[7,8] In our family 
the deleted fragment (c.-5413_-49) includes the entire P2 promoter region of the gene and segregated with the disease. All affected family members have typical features of peripheral neuropathy with varying severity suggesting absence of $\mathrm{P} 2$ in causing pathogenic nerve dysfunction. Male members reported early onset of symptoms. None of the affected individuals had hip dysplasia which is an association described with CMT.[11] They did not have central nervous involvement as reported with some X linked CMT families.[13,16] Electrophysiology was consistent with peripheral nerve dysfunction and nerve biopsy in one individual excluded inflammatory cause of neuropathy.

$\mathrm{P} 2$ promoter region is important for gene function as reduced or absent (as in our family) activity will cause a reduction/ loss of messenger RNA transcript, hence reduced GJBI protein.[9] Functional analysis of promoter region by Houlden et al. (2004) identified its role to cause Schwann cell dysfunction by interacting with SOX10 and EGR2 genes.[9] Interaction between them is complex. SOX10 and EGR2 bind to the P2 promoter region of GJB1 gene at S1-S2 and E2-E3 sites respectively for transcription.[17,18] This interaction between GJB1 P2 promoter, SOX10 and EGR2 can be interrupted by either mutations in SOX10[19] and EGR2[20] genes or mutation in P2 promoter region of GJB1.[9] Our patients did not have mutations in SOX10 and EGR2 genes supporting restriction of transcription by latter mechanism. This observation strengthens the hypothesis by Houlden et al. that P2 promoter is central in function of Schwann cell in peripheral nerve.

\section{Conclusion}

We have described a novel mutation deleting entire $\mathrm{P} 2$ promoter of $G J B 1$ gene in a single large family with CMT. Inheritance and phenotype of affected individuals had classical features of X linked peripheral neuropathy. This study affirms the role of P2 promoter being vital for Schwann cell function. 


\section{References}

[1] Bergoffen J, Scherer SS, Wang S, et al. Connexin mutations in X-linked CharcotMarie-Tooth disease. Science 1993, 262:2039-42.

[2] Pareyson D, Marchesi C. Diagnosis, natural history, and management of Charcot-MarieTooth disease. Lancet Neurol 2009, 8:654-67.

[3] Scherer SS, Deschenes SM, Xu YT, et al. Connexin 32 is a myelin- related protein in the PNS and CNS. J Neurosci 1995,15: 8281-94.

[4] Söhl G, Gillen C, Bosse F, et al. A second alternative transcript of the gap junction gene connexin 32 is expressed in murine Schwann cells and modulated in injured sciatic nerve. Eur J Cell Biol 1996, 69:267-75.

[5] Neuhaus IM, Dahl G, Werner R. Use of alternate promoters for tissue-specific expression of the gene coding for connexin 32. Gene 1995,158: 257-62.

[6] Neuhaus IM, Bone L, Wang S, et al. The human connexion 32 gene is transcribed from two tissue specific promotors. Biosci Reports 1996,16: 23948.

[7] Murphy SM, Polke J, Manji H, et al. A novel mutation in the nerve-specific 5'UTR of the GJB1 gene causes X-linked Charcot-Marie-Tooth disease. J Peripher Nerv Syst 2011, 16:65-70.

[8] Tsai PC, Chen CH, Liu AB, et al. Mutation analysis of the 5'non-coding region of GJB1 
in a Taiwanese cohort with Charcot-Marie-Tooth neuropathy. J Neurol Sci 2013, 332:515.

[9] Houlden H, Girard M, Cockerell C, et al. Connexin 32 promoter P2 mutations: a mechanism of peripheral nerve dysfunction. Ann Neurol 2004, 56:730-4.

[10] Li J, Lupat R, Amarasinghe K, et al. CONTRA: copy number analysis for targeted resequencing. Bioinformatics 2012, 28(10): 1307-13.

[11] Evans WH. Cell communication across gap junctions: a historical perspective and current developments. Biochem Soc Trans. 2015, 43(3):450-9.

[12] Bamford NS, White KK, Robinett SA, et al. Neuromuscular hip dysplasia in CharcotMarie-Tooth disease type 1A. Dev med Child Neurol 2009, 51(5): 408-11.

[13] Panas M, Kalfakis N, Karadimas C, et al. Episodes of generalized weakness in two sibs with the C164T mutation of the connexion 32 gene. Neurology 2001, 57:1906-8.

[14] Paulson HL, Garbern JY, Hoban TF, et al. Transient central nervous system white matter abnormality in X-linked Charcot-Marie-Tooth disease. Ann Neurol 2002, 52: 429-34.

[15] Hanemann CO, Bergmann C, Senderek J, et al. Transient recurrent white matter lesions in X-linked Charcot-Marie-Tooth disease with novel connexion 32 mutation. Arch Neurol, 2003, 60: 605-9.

[16] Taylor RA, Simon EM, Marks HG, et al. The CNS phenotype of X-linked CharcotMarie-Tooth disease: more than a peripheral problem. Neurology 2003, 61: 1475-8. 
[17] Boundurand N, Girard M, Pingault V, et al. Human Connexin 32, a gap junction protein altered in the X-linked form of Charcot-Marie-Tooth disease, is directly regulated by the transcription factor SOX10. Human Mol Genet 2011, 10:2783-95.

[18] Musso M, Balestra P, Bellone L, et al. The D355V mutation decreases EGR2 binding to an element within the Cx32 promoter. Neurobiol Dis 2001, 8:700-6.

[19] Inoue K, Tanabe Y, Lupski JR. Myelin deficiencies in both the central and the peripheral nervous system associated with a SOX10 mutation. Ann Neurol 1999, 46:313-8.

[20] Warner LE, Mancias P, Butler IJ, et al. Mutations in the early growth response 2 (EGR2) gene are associated with hereditary myelinopathies. Nat Genet 1998, 18:382-4. 